\title{
Sexprobleme: Konstruktiv kommunizieren hilft
}

Bei Patientinnen mit metastasiertem Brustkrebs können sexuelle Probleme die Lebensqualität zusätzlich beeinträchtigen. Das Risiko depressiver Symptome steigt - auch beim Partner. Unklar ist, welche Rolle das Kommunikationsmuster zwischen beiden dabei spielt.

\begin{abstract}
ie Diagnose Brustkrebs kann das Sexualleben erheblich belasten. Dazu tragen auch Vaginaltrockenheit, Stomatitis und atrophische Veränderungen als mögliche Folgen einer Chemotherapie bei. Aus Studien beim frühen Brustkrebs weiß man, dass sexuelle Probleme vermehrt mit depressiven Symptomen bei den Patientinnen und ihren Partnern einhergehen. Vergleichsweise wenig ist über diese Zusammenhänge beim metastasierten Brustkrebs bekannt und darüber, wie die Paare mit sexuellen Problemen zurechtkommen. Dem gingen Kathrin Milbury und Kollegen in einer Untersuchung bei 191 Patientinnen mit metastasiertem Brustkrebs unter Therapie und ihren Partnern nach. Besonderes Augenmerk galt dabei dem Kommunikationsmuster, das mithilfe eines Fragebogens (CPG $=$ Communication Patterns Questionnaire) erfasst wurde.
\end{abstract}

Die Patientinnen gaben signifikant mehr sexuelle Probleme an als ihre Partner. Die von jedem Paar getrennt ausgefüllten Fragebögen ergaben, dass das partnerschaftliche Kommunikationsmuster die Folgen in unterschiedlichem Maße beeinflusste. Ein signifikanter Zusammenhang zwischen erheblichen sexuellen Problemen und mehr depressiven Symptomen fand sich nur bei Patientinnen, die über wenig konstruktive Kommunikation $(\mathrm{p}<0,01)$ und viel durch Forderungs-/Rückzugsmuster geprägte Kommunikation $(\mathrm{p}<0,0001)$ berichteten. Dagegen reagierten ihre Partner unabhängig von dem angegeben Kommunikationsmuster vermehrt mit depressiven Symptomen auf die Probleme im Bett. Dieses Bild blieb auch bei Berücksichtigung der von den Patientinnen angegebenen Schmerzen, ihres funktionellen und körperlichen Wohl-

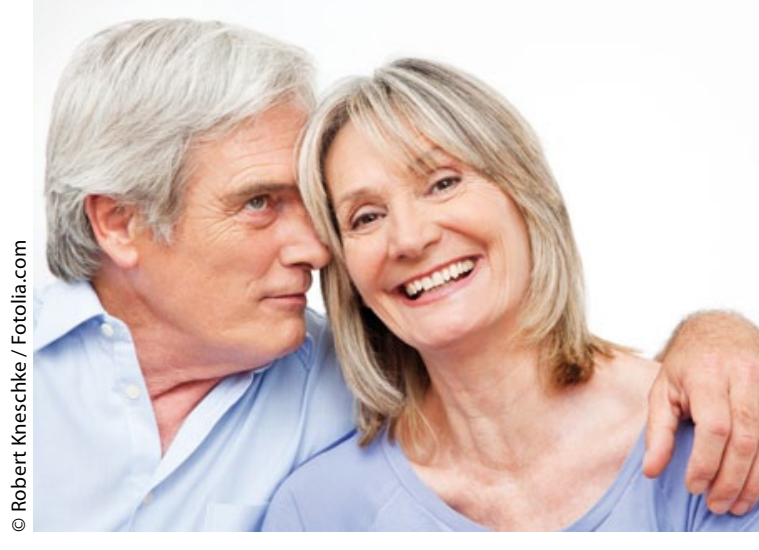

befindens sowie des Zustands der Paarbeziehung bestehen.

Fazit: Sexuelle Probleme gehen sowohl bei Patientinnen mit metastasiertem Brustkrebs als auch bei ihren Partnern mit depressiven Symptomen einher. Eine konstruktive Kommunikation scheint den Patientinnen aber einen Schutz vor der Entwicklung depressiver Symptome bieten zu können. Deshalb sollte man versuchen, einen offenen und konstruktiven Dialog der Paare über sexuelle Fragen zu fördern.

Ulrike Wepner

Milbury K et al. Sexual problems, communication patterns, and depressive symptoms in couples coping with metastatic breast cancer. Psychooncology 2012 [epub ahead of print]

\section{Dauerhaft helfen bei der Krankheitsbewältigung}

\section{Immer mehr Krebspatienten werden zu Langzeitüberlebenden. Viele von ihnen haben nicht nur mit körperlichen Symptomen zu kämpfen, sondern auch mit mentalen Problemen. Welche Rolle hier die Selbstwirksamkeit bei der Krankheitsbewältigung spielt, ist unklar.}

$B^{c}$ ei schätzungsweise $30 \%$ der Krebspatienten muss in der Behandlungsphase mit erheblichem psychologischem Distress gerechnet werden. Die mentalen Bedürfnisse der Patienten finden in dieser Zeit inzwischen mehr Beachtung als früher. Dagegen weiß man noch relativ wenig darüber, wie es später weitergeht. Vor allem die Rolle möglicher protektiver Faktoren, wie Selbstwirksamkeit bei der Krankheitsbewältigung und soziale Unterstützung, ist nicht geklärt.

Eine US-Arbeitsgruppe ging diesen Fragen jetzt bei 124 Krebsüberlebenden, davon gut die Hälfte mit Brustkrebs, nach. Das mittlere Alter betrug 62,2 Jahre, die Behandlung lag durchschnittlich 9,3 Jahre zurück. Zur Erfas- sung von körperlichen Symptomen, Selbstwirksamkeit bei der Krankheitsbewältigung, sozialer Unterstützung und Depression füllten sie verschiedene Fragebögen aus.

Bei etwa jedem Fünften gab es Hinweise auf eine klinisch relevante Depression. Die Selbstwirksamkeit bei der Krankheitsbewältigung erwies sich dabei als hoch signifikanter Prädiktor, der für $43 \%$ der Varianz hinsichtlich depressiver Probleme verantwortlich war. Vor allem zwei Teilbereiche schienen bedeutsam: Vertrauen in die Steuerung von Emotionen sowie in den Erhalt der Aktivität. Auch im Hinblick auf die Beziehung zwischen Einfluss vorhandener Symptome und Auftreten einer Depres- sion spielte die Selbstwirksamkeit bei der Krankheitsbewältigung zumindest partiell eine Rolle. Dagegen war der Faktor soziale Unterstützung von untergeordneter Bedeutung und kein signifikanter Prädiktor für Depression (verantwortlich für $9 \%$ der Varianz).

Fazit: Eine beträchtlicher Teil der Krebsüberlebenden zeigt noch viele Jahre nach Abschluss der Behandlung klinisch relevante Symptome einer Depression. Eine wichtige Rolle in diesem Zusammenhang scheint die Selbstwirksamkeit bei der Krankheitsbewältigung zu spielen. Sie sollte durch gezielte Intervention gefördert werden. Die Ergebnisse dieser Studie machen auch deutlich, dass Krebsüberlebende auf Dauer Unterstützung hinsichtlich ihrer seelischen Gesundheit benötigen. Ulrike Wepner

Philip EJ et al. Depression and cancer survivorship: Importance of coping self-efficacy in posttreatment survivors. Psychooncology. 2012.

[Epub ahead of print] 